\title{
Polyamine Analogue
}

National Cancer Institute

\section{Source}

National Cancer Institute. Polyamine Analogue. NCI Thesaurus. Code C49109.

A compound that mimics the naturally occurring polyamines but is modified in such a way that its function differs from that of the natural polyamines and is usually biologically inactive. Polyamine analogues most likely exert their actions through their ability to displace the natural polyamines from their DNA binding sites. Since the natural polyamines are involved in numerous important cellular processes such as cell division, differentiation and membrane function, their displacement from the DNA binding site may lead to an inhibition of cell growth. 